\title{
NUMERICAL CALCULATION ALGORITHM OF ATTITUDE AND MOVEMENT OF A BOOMERANG
}

\author{
Pierre Bigot*, Valdemir Carrara*, Philipe Massad* \\ * National Institute for Space Research- INPE, Av. dos Astronautas, 1758 \\ São José dos Campos, SP, Brasil
}

Emails: pierre.bigot.01@gmail.com, val@den.inpe.br, philipemassad@hotmail.com

\begin{abstract}
Although boomerangs are known since more than 10000 years, the study of its explanation is quite recent. The objective of this work is to detail step by step how to get an algorithm which allows to simply compute the boomerang trajectory and attitude. In the literature, most of the time, a four wings boomerang is studied because of the simplification its symmetry induce. In this work, the "classic" two wings boomerang is studied. As the objective it to obtain an algorithm which can be used for numerical calculation, the mathematical development is done using the concept of vectrix, the matrix representation of vectors. To avoid numerical errors the attitude in terms of Euler angles will be integrated using the quaternions theory, exactly the same way it is done to calculate satellite attitude.
\end{abstract}

Keywords_ Boomerang, Non-linear Dynamic, Aerodynamic, numerical integration, quaternion

\begin{abstract}
Resumo - O Bumerangue é construído há mais de 10000 anos. Mesmo assim, a explicação física do seu movimento é bastante recente. O objetivo deste trabalho é detalhar passo a passo como ter um algoritmo que permite calcular de maneira simples a trajetória e atitude do bumerangue. Na literatura, a maior parte dos trabalhos considera um bumerangue de quatro pás, pois a simetria induza uma simplificação das equações. Neste trabalho, o "clássico"bumerangue com duas pás é estudado. Como o objetivo é obter um algoritmo que possa ser utilizado para cálculos numéricos, o desenvolvimento matemático fará uso das vetrizes; a representação matricial dos vetores. O movimento rotacional em termos de ângulo de Euler será integrado usando a teoria dos quatérnions, da mesma maneira que é feito para calcular a atitude de satélite.
\end{abstract}

Palavras-chave - Bumerangue, Dinâmica não linear, Aerodinâmica, integração numérica, quatérnion

\section{Introduction}

The objective of this work is to create and algorithm which permits to calculate the boomerang flight: position and attitude at each instant. Then, this algorithm has to be tested with a numerical tool to validate the algorithm.

Let's first analyse the reasons that make the boomerang to return to its initial point. A simple explanation of this phenomenon has been written by Hunt (2001). The boomerang airfoil generates aerodynamics forces proportional to the incident air flux velocity. As the boomerang is simultaneously spinning and translating the incident air flux is different on the two boomerang wings. It results in aerodynamics lift forces different on the two wings, creating o moment which makes the boomerang to lay down. Then, due to the rotation inertia, this laying movement creates a gyroscopic precession and makes the boomerang plan to rotate to the left side of the throwing direction. To analyze boomerang movement, it will be needed to calculate the boomerang inertia, define a boomerang frame in order to calculate the boomerang attitude, get the kinematic and dynamic equations to both position and attitude computation and finally to calculate the aerodynamic forces and moments.

\section{Boomerang model}

Figure (1) shows a boomerang representation. It is a quite simple model consisting of only two wings. The boomerang parameters are the following: wing length $L$, wing width $l$, thickness $e$ and surface density $\sigma$. It will be considered that the boomerang is flat, which means that $e<<l$ and $e<<L$. Besides, $e$ is considered as a mean value, since the thickness depends on the wing airfoil shape. $R_{i}=(x, y, z)$ is defined as the boomerang reference frame, centered in $G$, the boomerang center of gravity. Because of boomerang symmetry and the small thickness, $b$ is the only distance which characterize the gravity center position relative to the boomerang body. This distance can easily be found with

$$
b=\frac{\sigma}{2 m}\left(l L^{2}+3 L l^{2}+l^{3}\right)-l
$$

\section{Boomerang inertia}

The boomerang inertia matrix $I$ can be easily calculated by directanalytical integration, if one assumes that the thickness is constant over the wing lenght and width. Let $\overrightarrow{G P}$ be the vector of a current point $P$ on the boomerang. Its coordinates axis expressed in the boomerang referential is $r=\left[\begin{array}{lll}x & y & 0\end{array}\right]^{T}$ as the boomerang is considered totally flat. The inertia matrix is calculated in the gravity center $G$ according to the $R_{i}$ axis. The 


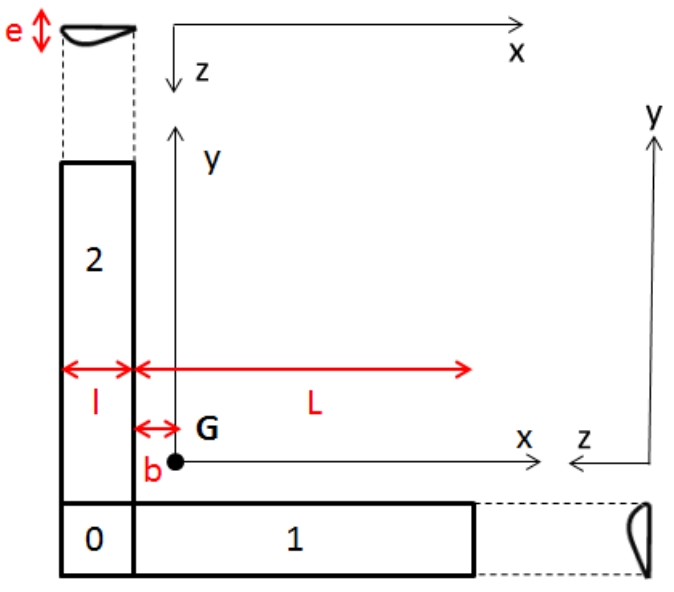

Figure 1: Boomerang representation

inertia matrix can be divided in two parts, $I_{0+1}$ considering the wing along the $x$ axis and $I_{2}$ considering the second wing. Equation (2) gives the inertia matrix expression. This integral has been computed using Matlab and its symbolic calculation tool, avoiding unnecessary hand calculation and leaving the expression more generic. Thus computing the inertia matrix of other boomerang shapes is easily done adding few modifications.

$$
\begin{aligned}
I_{0+1} & =\sigma \int_{x=-b-l}^{-b+L} \int_{y=-b-l}^{-b} r^{T} r-r r^{t} \mathrm{~d} x \mathrm{~d} y \\
I_{2} & =\sigma \int_{x=-b-l}^{-b} \int_{y=-b}^{-b+L} r^{T} r-r r^{t} \mathrm{~d} x \mathrm{~d} y \\
I & =I_{0+1}+I_{2}
\end{aligned}
$$

With a boomerang mass of $0.075 \mathrm{~kg}$, length of $0.4 \mathrm{~m}$ and width of $0.035[\mathrm{~m}]$, one gets the inertia matrix

$$
I=10^{-4}\left[\begin{array}{ccc}
14 & 8 & 0 \\
8 & 14 & 0 \\
0 & 0 & 27
\end{array}\right]
$$

\section{Euler angles}

To describe the movement the boomerang need to be located in the inertial frame $R_{i}=(X, Y, Z)$. Euler angles of 3-1-3 rotation $(\mathrm{Z}, \mathrm{X}, \mathrm{Z})$ is well adapted to this problem. Let be Euler angles $\beta, i$ and $\theta$. These angles can be seen in the Figure (2). Physically, $\beta$ represents the boomerang throwing direction in the plan $(X, Y), i$ its inclination with respect to the horizon plan and $\theta$ the spin angle.

\section{Kinematics and dynamics}

With defined referentials, it is now possible to express the boomerang motion equations. The rotational movement will be integrated in the boomerang fixed body frame $R_{b}$. As stated in
Huges (1986) and Carrara (2012), for the kinematic equations, the use of quaternions theory leads to a simple expression for the angular rates of the boomerang referential with respect to the inertial frame.

$$
\dot{Q}=\frac{1}{2} \Omega Q
$$

where $Q$ is the quaternion representation of euler angles $(\beta, i, \theta)$, and $\Omega$ is defined by

$$
\Omega=\left[\begin{array}{cc}
-\omega & \omega \\
-\omega^{T} & 0
\end{array}\right]
$$

and $\omega=\left[\begin{array}{lll}\omega_{1} & \omega_{2} & \omega_{3}\end{array}\right]^{T}$ is the boomerang angular rate. The operator ${ }^{\times}$stands for the matrix cross product defined as

$$
x^{\times}=\left[\begin{array}{ccc}
0 & -x_{3} & x_{2} \\
x_{3} & 0 & -x_{1} \\
-x_{2} & x_{1} & 0
\end{array}\right]
$$

The quaternions advantage is that there are no numerical singularity or trigonometric computation problems unlike using Euler angles. This equation can be integrated numerically in terms of quaternions. In order to analyze the result physically, the quaternion has to be transformed in $\mathrm{Eu}-$ ler angles. These angles can be extract from the rotation matrix $R b i$ which allows to pass from the inertia frame to the boomerang body frame. The relation between rotation matrix and quaternion is

$$
R_{b i}=\left(\eta^{2}-\epsilon^{T} \epsilon\right) I_{3}+2 \epsilon \epsilon^{T}-2 \eta \epsilon^{\times}
$$

where $\eta$ is the quaternion scalar part, $\epsilon$ its vectorial part and $I_{3}$ the identity matrix.

Finally the relation between Euler angles and the rotation matrix is

$$
R_{b i}=\left[\begin{array}{ccc}
c_{\theta} c_{\beta}-s_{\theta} c_{i} s_{\beta} & c_{\theta} s_{\beta}+s_{\theta} c_{i} c_{\beta} & s_{\theta} s_{i} \\
-s_{\theta} c_{\beta}-c_{\theta} c_{i} s_{\beta} & -s_{\theta} s_{\beta}+c_{\theta} c_{i} c_{\beta} & c_{\theta} s_{i} \\
s_{i} s_{\beta} & -s_{i} c_{\beta} & c_{i}
\end{array}\right]
$$

addopting the notation $c_{x}=\cos (x)$ and $s_{x}=$ $\sin (x)$. This relation can also be inverted to extract Euler angles:

$$
\begin{aligned}
\beta & =\arctan \left(\frac{r_{31}}{-r_{32}}\right) \\
i & =\arccos \left(r_{33}\right) \\
\theta & =\arctan \left(\frac{r_{13}}{r_{23}}\right)
\end{aligned}
$$

with $r_{k l}$ the term line $k$ and column $l$ of the $\operatorname{matrx} R_{b i}$. These relations are valid for $i \in[0, \pi]$.

Huges (1986) shows that the dynamic equation for a rigid body rotating around its gravity center is 


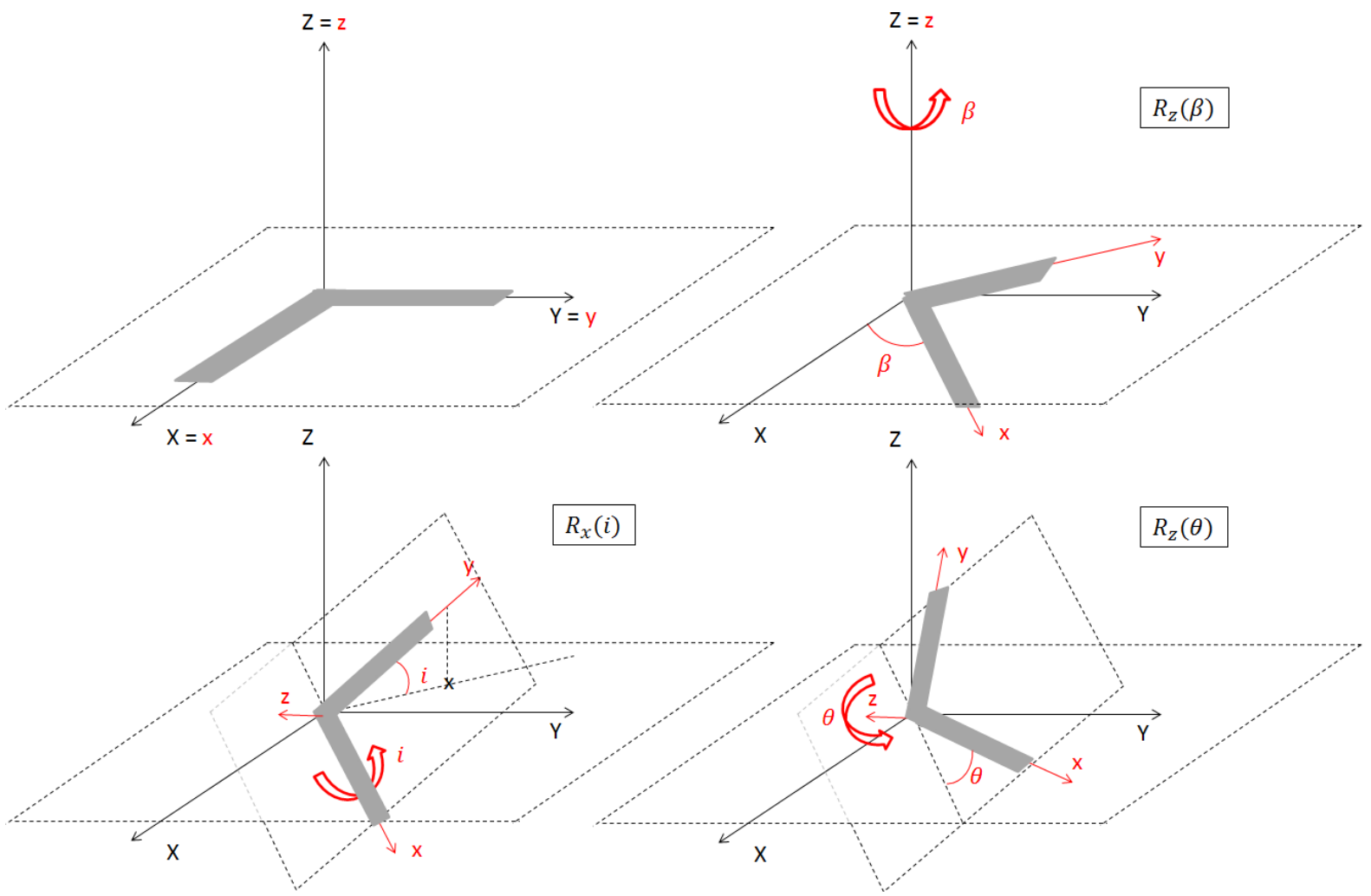

Figure 2: Euler angles $(\mathrm{Z}, \mathrm{X}, \mathrm{Z})$

$$
\dot{\omega}=I^{-1}\left(\Gamma_{G}-\omega^{\times} I \omega\right)
$$

where $\Gamma_{G}$ is the sum of external torques applied to the boomerang.

Finally, the translational motion will be integrated in the inertial frame. These equations are well known as Newton equations, where $F$ is the external resulting vector forces, $V_{G}$ is the boomerang gravity center speed and $P_{G}$ is the boomerang gravity center position in the inertial frame.

$$
\begin{aligned}
\dot{V}_{G} & =\frac{F}{m} \\
\dot{P}_{G} & =V_{G}
\end{aligned}
$$

The rotational equations are highly non-linear and there is no general analytic solution except for some few special configurations. Knowing external forces $F$ and torque $\Gamma_{G}$, Equations (4), (10) and (11) can be solved numerically to obtain the boomerang movement.

\section{Aerodynamics}

In this section, the external forces and torques on the boomerang will be obtained. The gavity force is easily calculated but aerodynamic effects need more attention. Let's consider a boomerang wing. Its airfoils induce lift and drag forces. It is well known that these forces are proportional to the air density $\rho$, to the planform area $S_{\text {ref }}$, to the true air speed squared $V_{T A S}^{2}$ and to the lift or drag coefficient $C_{L}$ and $C_{D}$. The aerodynamic forces expressions are

$$
\begin{aligned}
F_{L} & =\frac{1}{2} \rho V_{T A S}^{2} S_{r e f L} C_{L} \\
F_{D} & =\frac{1}{2} \rho V_{T A S}^{2} S_{r e f D} C_{D}
\end{aligned}
$$

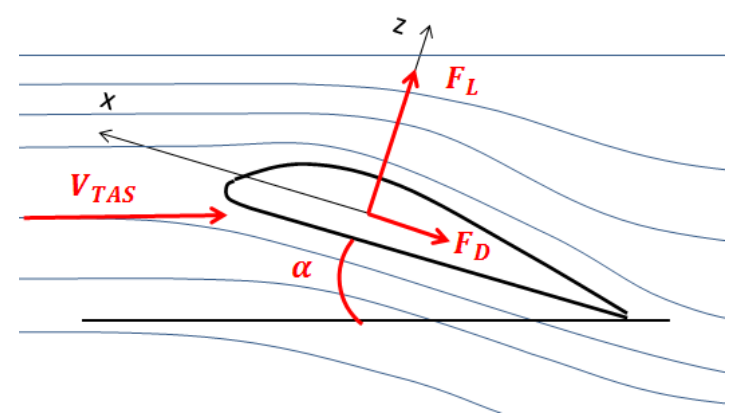

Figure 3: Airfoil in an air flux

The planform area can be considered as the boomerang projection in the plan $(x, y)$ for the lift surface and in the plan $(y, z)$ for the drag coefficient. Hence, in this study $S_{r e f L}=L l$ and $S_{\text {refD }}=l e$.

Both lift and drag forces are in the airfoil plan and as illustred in the Figure (3), the lift 
force $F_{L}$ is perpendicular to the air flux and the drag force is collinear to the incident air flux.

The true air speed $V_{T A S}$ is the relative speed between the air and the wing. For the boomerang motion take place it has to be considered the translation speed of its center of gravity $V_{G}$ and its rotational spin speed $\omega_{z}=\dot{\theta}$. As see in Figure (4), wings speeds are function of the distance from the center of gravity to the current point. Wings true air speeds $V_{T A S 1}$ and $V_{T A S 1}$ are exactly the oposite of the wings speeds. These speeds are given by

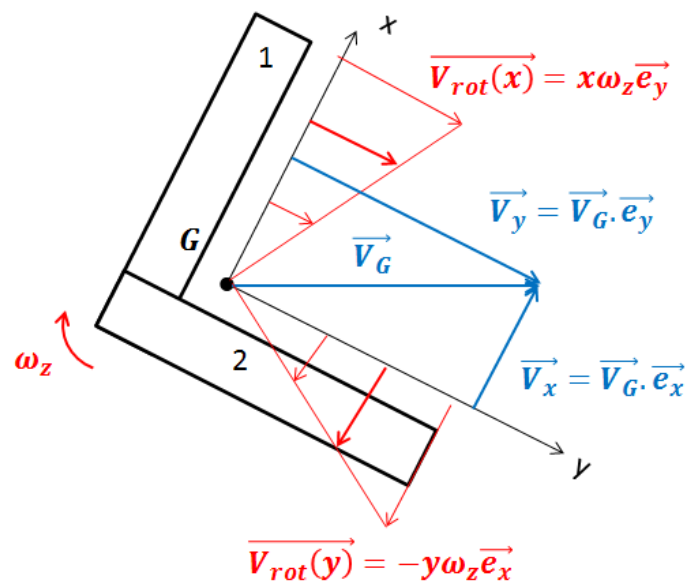

Figure 4: Boomerang different speeds representation

$$
\begin{aligned}
& V_{T A S 1}=-\left(x \omega_{z}+V_{y}\right) \\
& V_{T A S 2}=-\left(-y \omega_{z}+V_{x}\right)
\end{aligned}
$$

The angle of attack $\alpha$ is the angle between the boomerang speed vector $V_{G}$ and its projection on the boomerang plan $(x, y)$ as illustrated in the Figure (5). The attack angle can be expressed in function of the boomerang speed:

$$
\sin (\alpha)=\frac{V_{z}}{\sqrt{V_{G}^{T} V_{G}}}
$$

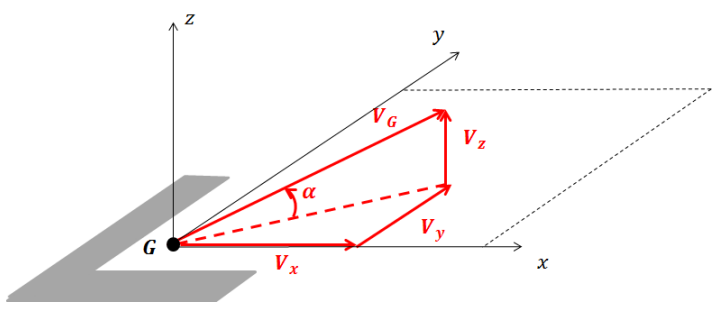

Figure 5: Attack angle representation

It is well known that the lift force increase with this angle. It is due to the dependency of the lift coefficient $C_{L}$ to the attack angle. According to Hess (1975), this dependency follows the relation

$$
C_{L}=C_{L 0}+2 \pi \sin (\alpha)
$$

where $C_{L 0}$ is the lift coefficient for an angle of attack null. In this study it will be considered that this attack angle stay little and then that the lift and drag coefficients are constant.

Finally, as the rotational speed is not constant along the chord, it is needed to integrate these speeds along the chord in order to get the total lift and drag force. The sustentation created by the central part of the wing (i.e. $x<0$ for wing 1 and $y<0$ for wing 2) has been neglect:

$$
\begin{aligned}
& F_{L 1}=C_{\text {aero }} \int_{x=0}^{L-b}\left(x \omega_{z}+V_{y}\right)^{2} \mathrm{~d} x \\
& F_{L 2}=C_{\text {aero }} \int_{y=0}^{L-b}\left(-y \omega_{z}+V_{x}\right)^{2} \mathrm{~d} y
\end{aligned}
$$

where $C_{\text {aero }}$ is defined as

$$
C_{\text {aero }}=\frac{1}{2} \rho C_{L} L l
$$

These integrals which represent the lift forces on airfoils 1 and 2 results in

$$
\begin{aligned}
& F_{L 1}=C_{\text {aero }} \frac{\left(\omega_{z}(L-b)+V_{y}\right)^{3}-V_{y}^{3}}{3 \omega_{z}} \\
& F_{L 2}=C_{a e r o} \frac{\left(\omega_{z}(L-b)-V_{x}\right)^{3}+V_{x}^{3}}{3 \omega_{z}}
\end{aligned}
$$

Expressions for drag forces $F_{D 1}$ and $F_{D 2}$ are easily obtained by substituting $C_{L} L l$ by $C_{D} l e$.

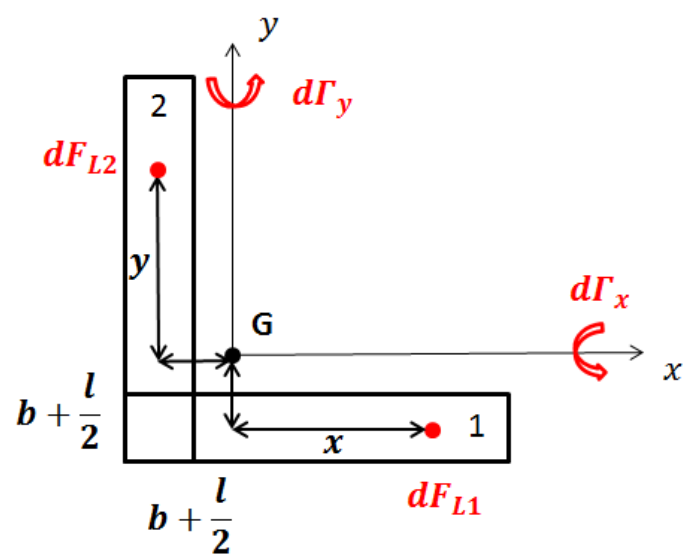

Figure 6: Boomerang resulting torques

As these forces are not exerced on the gravity center, they generate a torque. Both $F_{L 1}$ and $F_{L 1}$ generates torques along the axis $x$ and $y$. Figure (6) shows the representation of an elementars torques due to elementar lilt forces on the wing. It 
is considered that the force application is at $l / 2$ of the wing width but an application point along the wing length is unknown. Then, ones need to integrate along the wing length to obtain the resulting torques:

$$
\begin{aligned}
\Gamma_{x} & =C_{\text {aero }} \int_{y=0}^{L-b} y\left(-y \omega_{z}+V_{y}\right)^{2} \mathrm{~d} y \\
& -F_{L 1}\left(b+\frac{l}{2}\right) \\
\Gamma_{y} & =C_{\text {aero }} \int_{x=0}^{L-b} x\left(x \omega_{z}+V_{x}\right)^{2} \mathrm{~d} x \\
& +F_{L 2}\left(b+\frac{l}{2}\right)
\end{aligned}
$$

These integrals results in

$$
\begin{aligned}
\Gamma_{x} & =C_{\text {aero }}\left(\frac{L_{b}^{4} \omega^{2}}{4}+\frac{2 L_{b}^{3} \omega V_{y}}{3}+\frac{L_{b}^{2} V_{y}^{2}}{2}\right) \\
& -F_{L 1}\left(b+\frac{l}{2}\right) \\
\Gamma_{y} & =C_{\text {aero }}\left(\frac{L_{b}^{4} \omega^{2}}{4}-\frac{2 L_{b}^{3} \omega V_{x}}{3}+\frac{L_{b}^{2} V_{x}^{2}}{2}\right) \\
& +F_{L 2}\left(b+\frac{l}{2}\right)
\end{aligned}
$$

with $L_{b}=L-b$.

\section{Calculation algorithm}

The final algorithm is summarized in Figure (7). At each step, based on Equation (7), the function quatrmx returns the quaternions representation giving euler angles $(Z, X, Z)$ and the function quatezxz which use Equations (7) and (8) allows physicaly representation of what is happening, returning euler angles $(Z, X, Z)$ giving the quaternion. Notation (1), (2) or (3) refers to the first second or last component of the concerned vector. The Figure (8) summarizes equations to be integrated.

\section{Simulation results}

The boomerang motion has been simulated with Matlab. Typical initial conditions has been found in Hess (1975) and has been sumarized in Table (1). The boomerang is launched in the direction $X$ of the inertial frame with an angle of $20 \mathrm{deg}$ above the horizon and with $25 \mathrm{~m} \mathrm{~s}^{-1}$ velocity magnitude. The boomerang axis $y$ is aligned with the inertial axis $X$ and the boomerang plan is inclined of 10 deg from the inertial plan $(X, Y)$.

With boomerang characteristics exposed in the Table (2) a trajectory almost circular is obtained. From the "standard" configuration of Table (1), initial conditions have been slightly modified in order to see their effects on the boomerang

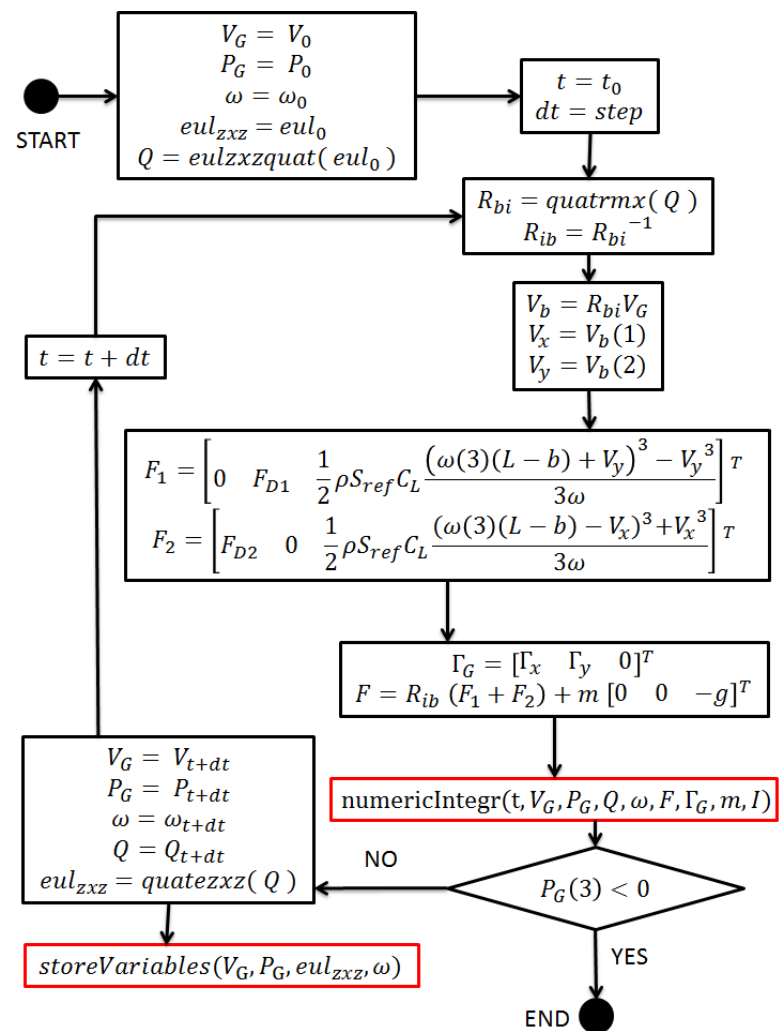

Figure 7: Global algorithm for boomerang atitude and position calculation

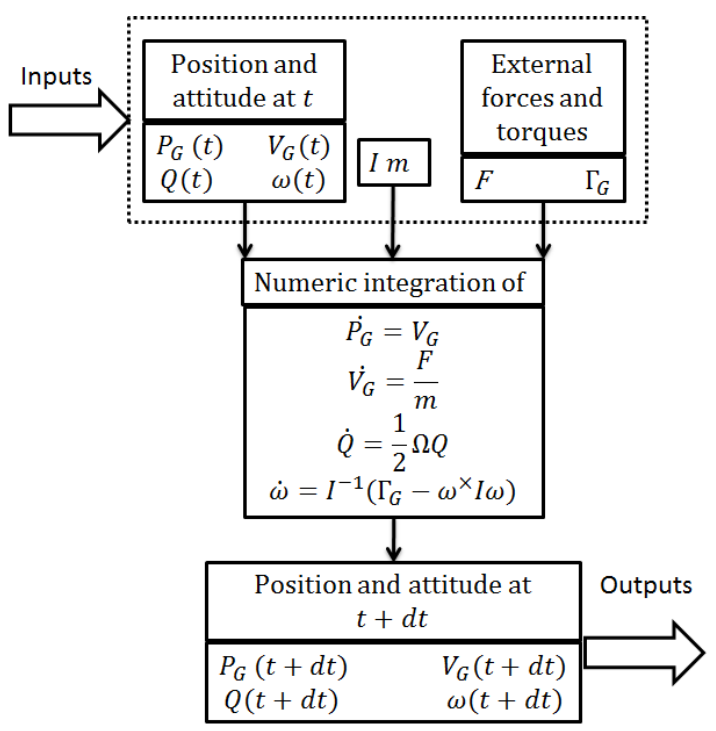

Figure 8: Numerical integration inputs and outputs

\begin{tabular}{c|c} 
Initial conditions & values \\
\hline$P_{0}[\mathrm{~m}]$ & {$\left[\begin{array}{lll}0 & 0 & 2\end{array}\right]^{T}$} \\
$V_{0}\left[\mathrm{~m} \mathrm{~s}^{-1}\right]$ & {$\left[\begin{array}{lll}23.5 & 0 & 8.6\end{array}\right]^{T}$} \\
$\omega_{0}\left[\mathrm{rad} \mathrm{s}^{-1}\right]$ & {$\left[\begin{array}{lll}0 & 0 & 62.8\end{array}\right]^{T}$} \\
$e u l_{z x z}[\mathrm{deg}]$ & {$\left[\begin{array}{lll}180 & 80 & 90\end{array}\right]^{T}$}
\end{tabular}

Table 1: Initial conditions 


\begin{tabular}{c|c} 
Boomrerang & Values \\
\hline$L[\mathrm{~m}]$ & 0.4 \\
$l[\mathrm{~m}]$ & 0.035 \\
$\sigma\left[\mathrm{kg} \mathrm{m}^{-2}\right]$ & 2.56
\end{tabular}

Table 2: Boomerang characteristics

trajectory. Figure (9) shows the boomerang trajectory for the standard configuration in blue and for a: bigger lift coefficient in green, lower initial speed angle in red and lower angle $i$ in cyan. When the lift coefficient is bigger, the trajectory becomes tight. When the initial speed angle is lower, the boomerang doesn't reach the same altitude. Finally when the inclination $i$ is lower, the boomerang goes high in the sky before going down. This inclination angle is the angle between the boomerang plan and the horizon plane. Thus it is easy to see that for a small value of $i$, the boomerang goes higher because the lift force would mainly be directed in the upper direction (axis $Z$ ).

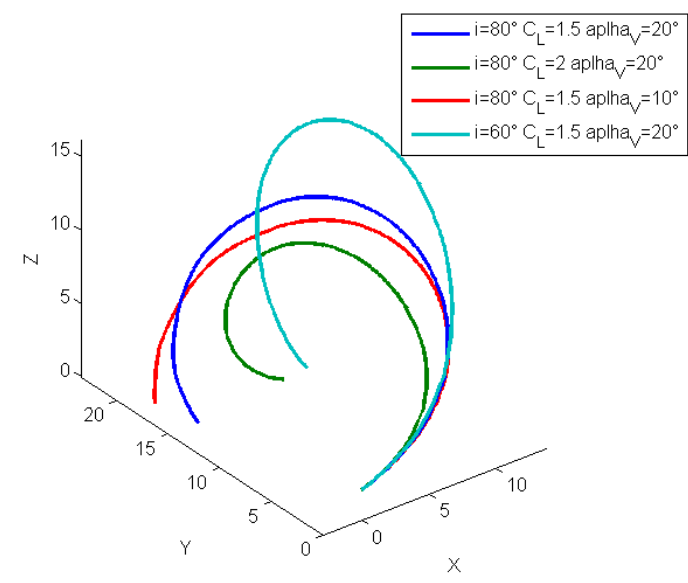

Figure 9: Boomerang trajectories for different initial parameters

These trajectories last more or less $4 \mathrm{~s}$. In the fourth case it reach an altitude of almost 16 $\mathrm{m}$ whereas it reach only $7 \mathrm{~m}$ in the third case. Considering the distance reached in the horizontal plane, in the fourth case the boomerang reach more than $24 \mathrm{~m}$ whereas in the second case it reached only $15 \mathrm{~m}$.

For none of these configurations, the boomerang went back exactly to the initial point. As it has be seen on the different curves, the trajectory is really different for slight initial condition modifications. That's why to get such result it would be needed to test a lot of different configurations.

\section{Conclusion}

The objective of this work was to simulate the motion of a boomerang flight. It has been shown that the use of the quaternion theory greatly simplified the numerical process because they were no need to take care about special angle conditions during the integration process. This study has shown that different parameters can influence the boomerang flight. In the result section it has been seen that initial condition influence greatly boomerang behavior. The advantage of the simulator is to be able to test many initial conditions.

\section{Acknowledgements}

We are thankfull for the Brasilian organization CAPES for the master degree scholarship.

\section{References}

Carrara, V. (2012). Cinemática e dinâmica de satélites artificiais.

Hess, F. (1975). Boomerangs, Aerodynamics, and Motion, PhD thesis, University of Groningen.

Huges, P. C. (1986). Spacecraft Attitude Dynamics, Doever.

Hunt, H. (2001). Boomerang theory. 